\title{
WEED DETECTION BASED ON THE OPTIMIZED SEGMENTATION LINE OF CROP AND WEED
}

\author{
Wenhua Mao, Xiaoan Hu, Xiaochao Zhang * \\ Institute of Mechatronis Technology and Application, Chinese Academy of Agricultural \\ Mechanization Sciences, Beijing, China, 100083 \\ * Corresponding author, Address: P.O. Box 121, Institute of Mechatronis Technology and \\ Application, Chinese Academy of Agricultural Mechanization Sciences, No. 1, Bei Shatan, \\ Dewai, Beijing, 100083, P. R. China, Tel: +86-10-64882584, Fax: +86-10-64882652, Email: \\ zxc@caams.org.cn
}

Abstract: Weed detection is a key problem of spot spraying that could reduce the herbicide usage. Spectral information of plants is very useful to detect weeds in real-time for the fast response time. However, the cost of an imaging spectrograph-based weed detection system is too high. Therefore, the main objective of this study was to explore a method to classify crop and weed plants using the spectral information in the visible light captured by a CCD camera. One approach to weed classification was to directly use of $G$ and $R$ component of RGB color space. Another was to utilize the spectral information among the green band that hue was regarded as wavelength, and saturation was represented as reflectance. The result of statistic analysis showed that both of them using the G-R and H-S optimized segmentation line of crop and weeds could be used to detect weed (lixweed tansymnustard) from wheat fields. Moreover, the method of using the H-S optimized model could avoid the affect of lighting.

Keywords: weed detection, color image, image process, spectrum information

\section{INTRODUCTION}

Research (Thompson et al., 1991; Yao et al., 1999) has shown that weeds are highly aggregated and that if herbicides were only applying over the weed-infected areas rather than uniform application, a reduction herbicide

Mao, W., Hu, X. and Zhang, X., 2008, in IFIP International Federation for Information Processing, Volume 259; Computer and Computing Technologies in Agriculture, Vol. 2; Daoliang Li; (Boston: Springer), pp. 959-967. 
usage would occur. This reduction has economical advantage and environmental benefit.

A weed-sensor and spray-control system (Tian, 2002) would facilitate spatially variable herbicide application. However, how to sensor the information of weed infestation in fields is a primary challenge for spot spraying. There are many method of weed detection, such as manual surveying (Stafford et al., 1996), remote sensing (Brown et al., 1995) and near ground-based approaches (Lee et al., 1999). The machine-vision based detection system is mainly used, because it has high spatial resolution sensors and may utilize spectral, spatial or texture information of plants.

Until now, shape feature of plant leaves has been successfully used to classify individual plants, or some plants with little occlusion leaves (Lee et al., 1999; Pérez et al., 2000). Weed detection used texture information of plant canopy can achieve a high rate of correction classification (Tang et al., 1999; Burk et al., 2000), but most recognition algorithms are timeconsuming for performed a lot of calculation and compared with cooccurrence matrices. Spectral approach imposes fewer restrictions on the response of time than other approaches (Robert et al., 2002), which greatly increases the potential for field implementation in a real-time spot spraying.

Borregaard et al. (2000) collected spectral data using line imaging spectroscopy in the 660 to $1060 \mathrm{~nm}$ spectral range. Features selected for the discriminant function classifiers were 694, 970, 856, 686, 726, 897 and $978 \mathrm{~nm}$. The correct classification was $90 \%$. A study using an imaging spectrograph was conducted by Vrindts and De Baerdemaeker (2000), over the spectral range of 485 to $815 \mathrm{~nm}$. Sugarbeet classifiers used up to 11 wavelength features, and corn up to 9 features. Feyaerts and van Gool (2001) developed an online weed sensor based on an imaging spectrograph. The spectral range of the system could be adjusted to cover interesting wavelengths $(441,446,459,883,924 \mathrm{~nm})$. Under field conditions, $86 \%$ of the vegetation samples were classified correctly.

However, the cost of an imaging spectrograph based weed detection system is too high, which limits its' application in the field of the Chinese farm. Therefore, the main objective of this study was to explore a method to classify crop and weed plants using the spectral information in the visible light captured by a CCD camera.

\section{SPECTRAL PROPERTIES OF GREEN PLANT}

The general reflectance properties of green vegetation have been well established (Fig. 1). It could be seen from the figure measured by Vrindts et al. (2000) that the mean reflectance of some green plants is different in the 


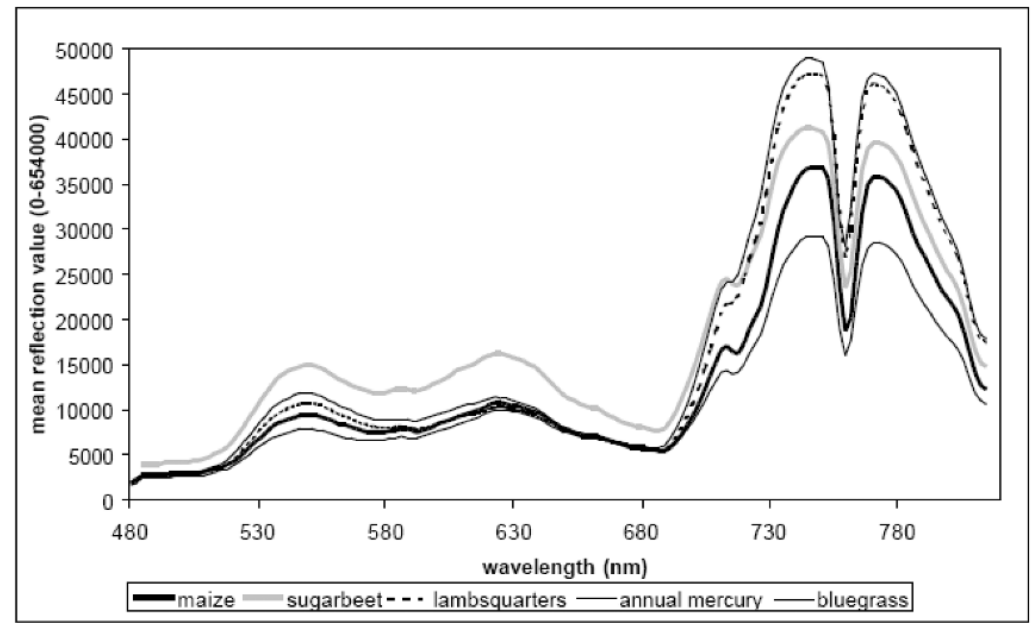

Fig. 1. Average spectra for maize, sugar beet and 3 weed species

green light (approximately 500-550nm). Moreover, there also has a difference in the red light (610-650nm).

Therefore, weed could be detected according to the difference of the mean reflectance among the visible light band. Color Images captured by a CCD camera can combine up to three bands (red, green and blue). Thus, one approach to weed classification was to directly use of $\mathrm{G}$ and $\mathrm{R}$ component of RGB color space. Another was to utilize the spectral information among the green band.

Color can be described by its wavelength. Hue is largely dependent on the dominant wavelength of light that is emitted or reflected from an object. Thus, a change in wavelength of visible light is manifested by a change in hue. Moreover, saturation depends upon the relative dominance of pure hue in a color sample. Hence, hue can be regarded as wavelength, and saturation can be represented as reflectance. The formula that converts from RGB to HS is described as follows:

$$
\left\{\begin{array}{l}
H=\cos ^{-1}\left[\frac{\frac{1}{2}[(R-G)+(R-B)]}{\sqrt{(R-G)^{2}+(R-B)(G-B)}}\right] \\
S=1-\frac{3}{R+G+B}[\min (R, G, B)]
\end{array}\right.
$$

If $\mathrm{B}$ is greater than $\mathrm{G}$, then $H=360^{\circ}-H$. 


\section{STATISTICAL ANALYSIS}

\subsection{Materials}

500 frames of color images were taken using a color digital camera (SONY Cyber Shot P7) in outdoor natural lighting conditions during 2005/2006 in the wheat fields of the China Agricultural University. The images were captured under various different conditions, such as the relative distance of camera and ground, the lighting (sunny or cloudy), the time (a.m. or p.m.), the stage of plant growth (3-5 leaves). The captured images included seedlings of wheat and weed (lixweed tansymnustard). Lixweed tansymnustard is a sort of dominant vicious weeds that often infests in winter wheat fields and severely affects the yield of wheat in China.

\subsection{Methods}

In order to find the useful color information of wheat and weed (flixweed tansymnustard), 500 frames collected color images were used for the statistical analysis as the follow steps:

\section{Step 1. Preprocess}

The original image included wheat plants, weed (flixweed tansymnustard) plants, soil and residue. The extra-green color index $(\operatorname{Exg}=2 \mathrm{G}-\mathrm{R}-\mathrm{B})$ was used to segment plants (keeping initial color) and background (transforming black $(0,0,0))$. Then, wheat plants and weed plants were manually extracted from the plant images (Fig. 2).

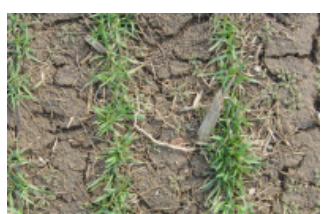

(a)

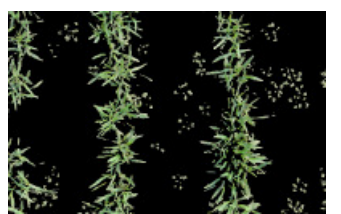

(b)

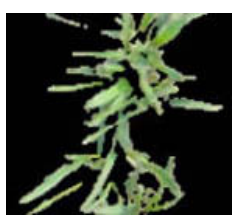

(c)

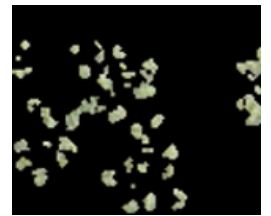

(d)

Fig. 2. The procedure of preprocess

(a) Original image; (b) Segmented plant image; (c) Extracted wheat image; (d) Extracted weed image

\section{Step 2. Data Distribution}

The G-R and H-S values of wheat and weed (flixweed tansymnustard) pixels were collected from the extracted plant images to build a G-R dataset and an H-S dataset, respectively. The software SPSS 10.0 for windows was used to statistically analyze two datasets of plant pixels. 
The simple scatter-plot was drawn to describe the original distribution of G-R or H-S. The scatter-plot graphs of two datasets were shown as Fig. 3. It could be seen that the distribution of G-R dataset was nearly linear, and that of $\mathrm{H}-\mathrm{S}$ dataset was curve.

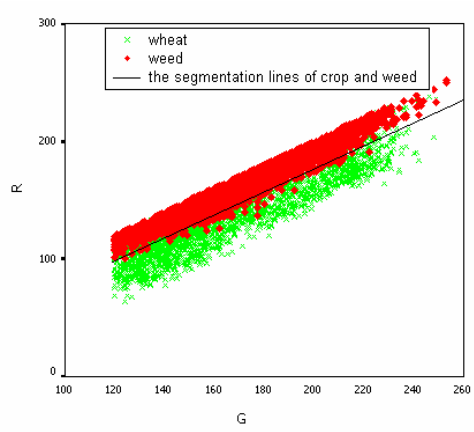

(a) G-R

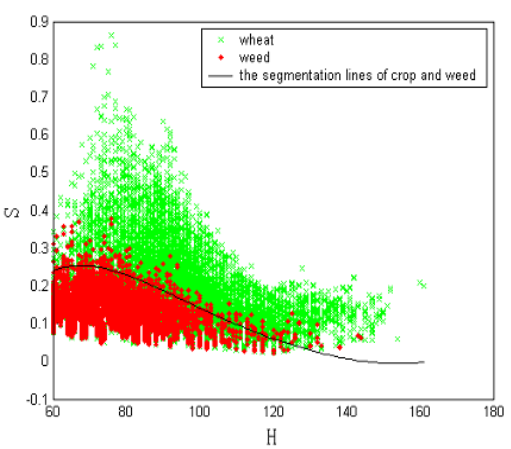

(b) $\mathrm{H}-\mathrm{S}$

Fig. 3. The scatter-plot graph

\section{Step 3. Discriminant Analysis}

The discrimination procedure was tested for the possibility of weed detection. The G-R and H-S data set were used to construct a discriminant function. The discriminant function, calculated with the enter independents together method is determined by a measure of generalized squared distance, based on the within-group covariance, yielding a quadratic discriminant rule. The built canonical discriminant fuctions were:

$$
\begin{aligned}
& \mathrm{D}=0.087 \mathrm{R}-0.075 \mathrm{G} \\
& \mathrm{D}=0.059 \mathrm{H}+5.799 \mathrm{~S}-6.758
\end{aligned}
$$

\section{Step 4. Regression Analysis}

To compress and centre the data, the value of the $\mathrm{R}$ dependent variable was replaced by its mean for each of the $\mathrm{G}$ independent variable. The H-S data were similarly processed. The regressive functions were shown in Table 1 , which were acquired by the linear and nonlinear regression procedure, respectively. The result of regression was shown in Fig. 4.

Table 1. The regression functions

\begin{tabular}{lll}
\hline & Regressive function & R2 \\
\hline G-R_crop & Rc=0.957G-22.078 & 0.992 \\
G-R_weed & Rw=0.994G-8.129 & 0.999 \\
H-S_crop & Sc=2E-06H3-0.0006H2+0.0619H-1.7474 & 0.871 \\
H-S_weed & Sw=7E-07H3-0.0002H2+0.0181H-0.3581 & 0.824 \\
\hline
\end{tabular}




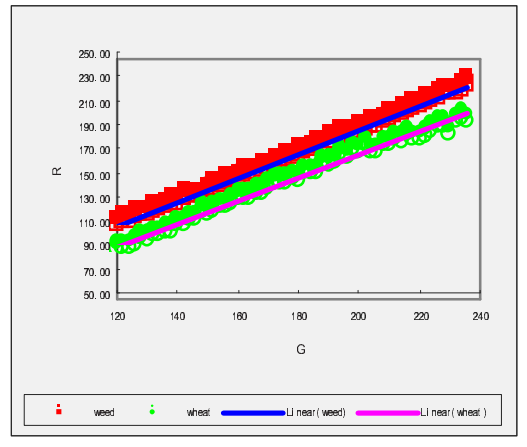

(a) G-R

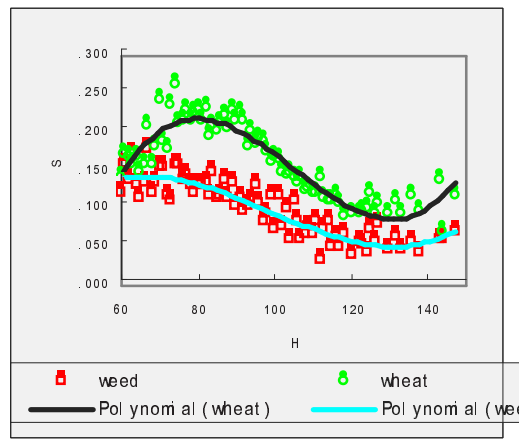

(b) H-S

Fig. 4. The scatter-plot graph after preprocess

Therefore, the G-R and H-S optimized models were as follows:

$$
\begin{aligned}
& R_{d}=R_{c}+k \frac{R_{w}-R_{c}}{2} \\
& S_{d}=S_{w}+k \frac{S_{c}-S_{w}}{2}
\end{aligned}
$$

\section{ALGORITHM DEVELOPMENT AND EVALUATION}

The algorithms based on the G-R and H-S discriminant and optimized models were developed using the software MS visual C++ 6.0 to segment wheat field color images into wheat and weed (flixweed tansymnustard). To decrease the effect of noise, the median filter of plant pixels was done in a $3 \times 3$ window. The pseudo codes of algorithms were as follows:

The first approach used the $\mathrm{G}$ and $\mathrm{R}$ component of RGB color space. For each plant pixel point $\left(\mathrm{R}_{\mathrm{i}}, \mathrm{G}_{\mathrm{i}}, \mathrm{B}_{\mathrm{i}}\right)$ computed as the followed formula:

$$
R_{d}=R_{c}+k \frac{R_{w}-R_{c}}{2}
$$

or

$$
\mathrm{D}=0.087 \mathrm{Ri}-0.075 \mathrm{Gi}-0.188
$$

If $\left(R_{i}>R_{d}\right.$ or $\left.D>0\right)$ then the plant pixel point was weed; else the plant pixel point was wheat.

The second method utilized the spectral information among the green band. For each plant pixel point $\left(\mathrm{R}_{\mathrm{i}}, \mathrm{G}_{\mathrm{i}}, \mathrm{B}_{\mathrm{i}}\right)$ computed $\mathrm{H}_{\mathrm{i}}$ and $\mathrm{S}_{\mathrm{i}}$ : 


$$
S_{d}=S_{w}+k \frac{S_{c}-S_{w}}{2}
$$

or

$$
\mathrm{D}=0.059 \mathrm{H}+5.799 \mathrm{~S}-6.758
$$

If $\left(S_{i}>S_{d}\right.$ or $\left.D>0\right)$ then the plant pixel point was wheat; else the plant pixel point was weed.

The detection results of using the discriminant function and optimized function were shown in Fig. 5. In the Fig. 5, the detected wheat pixels were green, and the classified weed pixels were red.

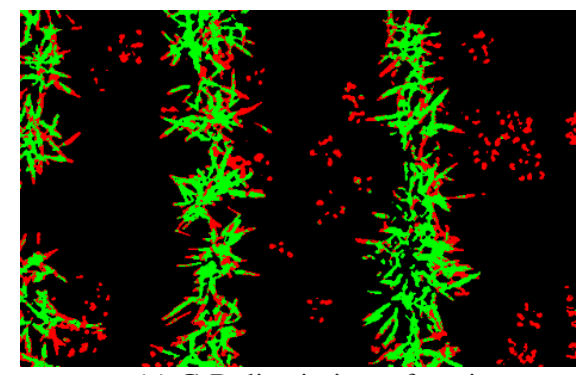

(a) G-R discriminant function

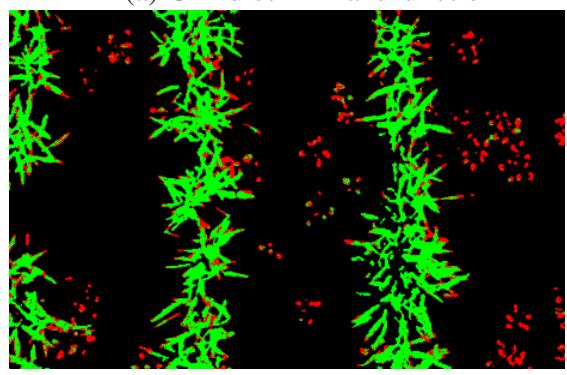

(c) G-R optimized function

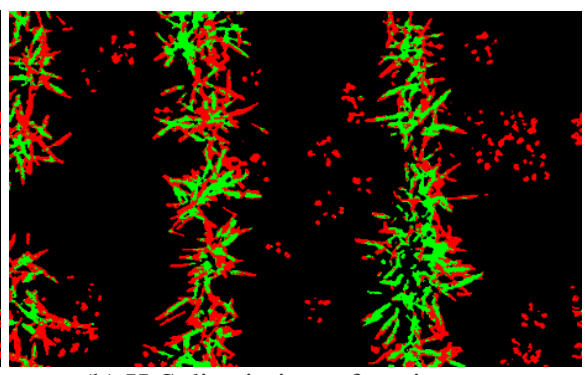

(b) H-S discriminant function

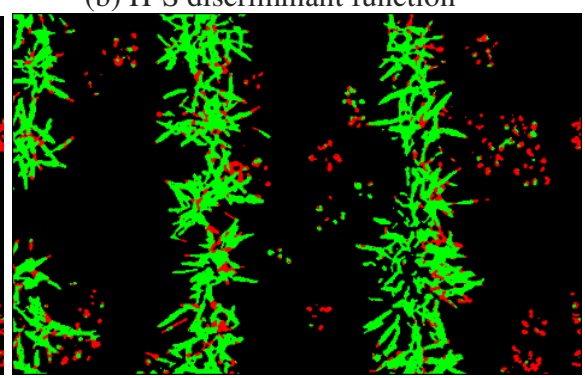

(d) H-S optimized function

Fig. 5. The classification results

\section{CONCLUSIONS}

For the 500 frames color images captured under the wheat fields, the means of correct classification using the optimized models were shown in Table 2.

It could be seen form the Table 2 that the optimized function greatly improved on the wheat correct classification, and basically held on the weed correct classification. 
Table 2. The classification results of using the optimized models

\begin{tabular}{lcc}
\hline & Weed correct classification & Wheat correct classification \\
\hline G-R dicriminant model & $92.5 \%$ & $75.3 \%$ \\
H-S dicriminant model & $90.6 \%$ & $56.7 \%$ \\
G-R optimized model & $91.4 \%$ & $87.6 \%$ \\
H-S optimized model & $90.1 \%$ & $88.2 \%$ \\
\hline
\end{tabular}

Therefore, both of approaches of using the G-R and H-S optimized model could be used to detect weed from wheat field. Moreover, the method of using the H-S optimized model could avoid of the affect of lighting. All of them only utilized the spectral information among the visible light, which would increase the feasibility of application under fields and decrease the cost of production.

\section{ACKNOWLEDGEMENTS}

This study has been funded by the National Natural Science Foundation of China (30500305).

\section{REFERENCES}

Borregaard T., H. Nielsen, L. Norgaard, et al., Crop-weed discrimination by line imaging spectroscopy, Journal of Agricultural Engineering Research, 2000, 75:389-400

Brown R. B., J.-P.G.A. Steckler, Prescription maps for spatially variable herbicide application in no-till corn, Transactions of the ASAE, 1995, Vol. 38, No. $6: 1659-1666$

Burks T.F., S.A. Shearer, F.A. Payne, Classification of weed species using color texture features and discriminant analysis, Transactions of ASAE, 2000, Vol. 43, No. 2:441-448

Feyaerts F., L. van Gool, Multi-spectral vision system for weed detection, Pattern Recognition Letters, 2001, 22:667-674

Lee W.S., D.C. Slaughter, D.K. Giles, Robotic Weed control system for tomatoes, Precision Agriculture, 1999, 1:95-113

Perez A.J., F. Lopez, J.V. Benlloch, et al., Color and shape analysis techniques for weed detection in cereal fields, Computers and electronics in agriculture, 2000, 25:197-212

Robert J. Baron, Trever G. Crowe, Thomas M. Wolf, Dual camera measurement of crop canopy using reflectance, AIC 2002 Meeting, CSAE/SCGR Program Saskatoon, July 1417,2002

Stafford J.V., J.M. Le Bars, B. Ambler, A hand-held data logger with integral GPS for producing weed maps by field walking, Computers and Electronics in Agriculture, 1996, 14:235-247

Tang L., L.F. Tian, B.L. Steward, et al., Texture-based weed classification using Gabor wavelets and neural network for real-time selective herbicide applications, ASAE, 1999, Paper No. 993036 
Thompson J.F., J.V. Stafford, P.C.H. Miller, Potential for Automatic Weed Detection and Selective Herbicide Application, Crop Protection, 1991, 10:254-259

Tian L., Development of a sensor-based precision herbicide application system. Computers and electronics in agriculture, 2002, 36:133-149

Vrindts E., J. De Baerdemaeker, Using spectral information for weed detection in field circumstances, Presented at AgEng 2000 Warwick, 2000, EurAgEng Paper No. 00-PA-010

Vrindts Els, Automatic recognition of weeds with optical techniques as basis for site-specific spraying, [Dissertations DE Agricultura]. Katholieke Universititeit Leuven, 2000

Yao H., L.F. Tian, L. Tang, et al., Smart sprayer performance simulation. ASAE, 1999, Paper No. 99-1103 